\title{
The Prefrontal Cortex Communicates with the Amygdala to Impair Learning after Acute Stress in Females but Not in Males
}

\author{
Lisa Y. Maeng, Jaylyn Waddell, and Tracey J. Shors \\ Department of Psychology and Center for Collaborative Neuroscience, Rutgers University, Piscataway, New Jersey 08854
}

\begin{abstract}
Acute stress exposure enhances classical eyeblink conditioning in male rats, whereas exposure to the same event dramatically impairs performance in females (Wood and Shors, 1998; Wood et al., 2001). We hypothesized that stress affects learning differently in males and females because different brain regions and circuits are being activated. In the first experiment, we determined that neuronal activity within the medial prefrontal cortex (mPFC) during the stressful event is necessary to disrupt learning in females. In both males and females, the mPFC was bilaterally inactivated with GABA agonist muscimol before the stressor. Inactivation prevented only the impaired performance in females; it had no consequence for performance in males. However, in the second experiment, excitation of the mPFC alone with GABA antagonist picrotoxin was insufficient to elicit the stress effect that was prevented through the inactivation of this region in females. Therefore, we hypothesized that the MPFC communicates with the basolateral amygdala to disrupt learning in females after the stressor. To test this hypothesis, these structures were disconnected from each other with unilateral excitotoxic (NMDA) lesions on either the same or opposite sides of the brain. Females with contralateral lesions, which disrupt the connections on both sides of the brain, were able to learn after the stressful event, whereas those with ipsilateral lesions, which disrupt only one connection, did not learn after the stressor. Together, these data indicate that the MPFC is critically involved in females during stress to impair subsequent learning and does so via communication with the amygdala.
\end{abstract}

\section{Introduction}

Traumatic life experiences tend to be more debilitating in women, rendering them twice as likely as men to develop stress and anxiety-related disorders (10.4 vs 5.0\%; Kessler et al., 1995; Carter-Snell and Hegadoren, 2003; Foa and Street, 2001; Tolin and Foa, 2006). This vulnerability in women may relate to sex differences in the stress response as reported in laboratory animals. For example, stressors such as inescapable swim stress or brief stimulations to the tail enhance a type of associative learning, classical eyeblink conditioning, in male rats and mice, whereas the same stressors elicit profound learning deficits in females (Wood and Shors, 1998; Wood et al., 2001). These behavioral differences in response to stress are mediated by sex differences in neural and hormonal processes within specific brain regions. The hippocampus and basolateral amygdala (BLA) are critically involved in both males and females to modify learning after stress (Bangasser and Shors, 2007; Waddell et al., 2008). However, the bed nucleus of the stria terminalis (BNST) is necessary only in males (Bangasser et al., 2005; Bangasser and Shors, 2008).

Received May 3, 2010; revised Sept. 22, 2010; accepted Sept. 30, 2010.

This work was supported by National Institutes of Health Grants 59970 and ARRA-59970-10S1 and National Science Foundation Grants IOB-044364 and I0S-0914386 (to T.J.S.).

Correspondence should be addressed to Tracey J. Shors, Department of Psychology and Center for Collaborative Neuroscience, Rutgers University, 152 Frelinghuysen Road, Piscataway, NJ 08854. E-mail: shors@rutgers.edu. DOI:10.1523/JNEUROSCI.2265-10.2010

Copyright $\odot 2010$ the authors $\quad 0270-6474 / 10 / 3016188-09 \$ 15.00 / 0$
Another likely participant is the medial prefrontal cortex $(\mathrm{mPFC})$, which is activated during the stress response and interconnects with the hippocampus, BNST, and BLA (Diorio et al., 1993; Vertes, 2004, 2006; Cerqueira et al., 2008). Stress-related disorders are associated with differences in both structure and function of the mPFC (Bremner et al., 1999; Drevets, 2000; Rajkowska, 2000; Luine, 2002). Furthermore, the female mPFC is especially sensitive to stress (Garrett and Wellman, 2009; Ter Horst et al., 2009) as well as to fluctuating estrogen concentrations (Gerrits et al., 2006). One study found that mPFC-mediated learning is more sensitive to stress in females than in males (Shansky et al., 2006). Therefore, we first hypothesized that the mPFC would be critically involved in females, but less so in males, to modify learning after a stressful experience. To test this hypothesis, the mPFC was either inactivated or activated during the stressor. One day later, both sexes were trained to learn the classically conditioned eyeblink response.

The mPFC and amygdala interconnect to affect emotional responses to stress, presumably via anatomical connections between them (Heidbreder and Groenewegen, 2003; Vertes, 2004). For example, lesions to the prefrontal cortex reduce the extinction of a fear response, which depends on the amygdala to learn (Morgan and LeDoux, 1995). Based on these interactions, we hypothesized that the mPFC and amygdala communicate with each other to reduce learning after stress, specifically in females. To test our hypothesis, both structures were excitotoxically lesioned on either the same (ipsilateral) or opposite (contralateral) 
sides of the brain. Those with ipsilateral lesions would have one intact connection, whereas those with contralateral lesions of the brain would have neither connection intact. As before, animals were either stressed or not and then trained $24 \mathrm{~h}$ later to learn the classically conditioned eyeblink response.

\section{Materials and Methods}

\section{Experiments 1 and 2: MPFC inactivation and activation}

Subjects. Male and cycling female Sprague Dawley rats between 90 and $120 \mathrm{~d}$ of age were obtained from a breeding facility at Rutgers University. Rats were housed in groups of 3-4 until surgery. Following surgery, rats were housed alone in standard plastic "shoebox" cages $(44.5 \mathrm{~cm}$ long, $21.59 \mathrm{~cm}$ wide, and $23.32 \mathrm{~cm}$ high). Rats were maintained on ad libitum access to rat chow and water on a $12 \mathrm{~h} \mathrm{light/dark} \mathrm{cycle.} \mathrm{All} \mathrm{experiments} \mathrm{were}$ conducted with full compliance to the rules and regulations specified by the Public Health Service (PHS) Policy on Humane Care and Use of Laboratory Animals and the Guide for the Care and Use of Laboratory Animals.

Vaginal cytology. To monitor the four phases of the estrus cycle, samples of loose vaginal cells were taken with cotton-tipped swabs soaked in sterile $0.9 \%$ saline and rolled onto slides (lavage). The slides were then stained with $1 \%$ toluidine blue, rinsed, and dehydrated with $95 \% \mathrm{ETOH}$ for estrus phase assessment under a light microscope. Proestrus is characterized by purple staining of epithelial cell nuclei, estrus by masses of aggregated dark blue cornified cells, diestrus 1 by dark leukocytes and scattered epithelial cells, and diestrus 2 by similar, but more sparse, cell types. It has been determined that the stress effect is most pronounced in females when they are stressed in diestrus 2 and trained $24 \mathrm{~h}$ later in proestrus (Shors et al., 1998). Therefore, females used in this study were lavaged daily after a 1 week recovery period following surgery, stressed in diestrus, and trained in proestrus, when estrogen concentrations were increasing (Shors et al., 1998; Wood et al., 2001). Animals that failed to exhibit a normal estrus cycle were eliminated from the study.

Surgery. All rats were anesthetized with sodium pentobarbital (50 $\mathrm{mg} / \mathrm{kg}$ for males and $40 \mathrm{mg} / \mathrm{kg}$ for females). After being placed in the stereotaxic instrument, the scalp was cleaned with Betadine, and an incision was made. Guide cannulas (23 gauge, Plastics One) were implanted into the mPFC bilaterally aimed at the junction of the prelimbic and infralimbic cortex. Cannulas were implanted at a $15^{\circ}$ angle at the following coordinates relative to bregma: (AP: $+3.1 \mathrm{~mm}$; ML: $\pm 1.6 \mathrm{~mm}$; DV: $-3.3 \mathrm{~mm}$ from dura). Following cannulation, both cannulas and headstages were fitted onto the skull with dental cement and anchored by skull screws. The headstages were attached to four electrodes; two delivered the unconditioned stimulus (US) of periorbital stimulation, and two recorded electromyographic (EMG) activity as a measure of blinks. The electrodes (insulated stainless steel wire with a diameter of 0.005 inches) were implanted through the upper eyelid muscle. The insulation was removed from a section of each electrode to make contact with the muscle. Each of the electrode wires was coiled securely in place.

Infusions. During microinfusions, stylets were replaced with infusion cannulas protruding $1 \mathrm{~mm}$ past the guide cannula. Infusion cannulas were attached to a microinfusion pump via polyethylene tubes connected to $10 \mu \mathrm{l}$ Hamilton syringes. The syringe and tubes were filled with water, and a small air bubble separated the water from the vehicle or drug solution. In experiment 1 , the $\mathrm{mPFC}$ was either infused with artificial CSF (aCSF) vehicle or bilaterally inactivated via infusions of $0.5 \mu \mathrm{g}$ of GABA-A receptor agonist muscimol $(1 \mu \mathrm{g} / \mu \mathrm{l})$ into each hemisphere. In experiment 2 , the $\mathrm{mPFC}$ was bilaterally activated via infusions of $0.5 \mu \mathrm{l}$ of either $100 \mathrm{ng}$ of picrotoxin (Sigma) or received microinjections of saline vehicle. All infusions were given at a rate of $0.125 \mu \mathrm{l} / \mathrm{min}$ over $4 \mathrm{~min}$ for a total of $0.5 \mu \mathrm{l}$.

Stress procedure. At least $7 \mathrm{~d}$ after the surgery, rats were acclimated to the conditioning chamber $(60 \mathrm{~min})$ and spontaneous blinks were recorded. They were then transported to a separate context and infused with aCSF or muscimol. Half of this group was transferred into another context (different from those in which the infusions and training occurred) and placed into a dark soundproof chamber. In this chamber, they were loosely restrained and exposed to 30 low-intensity ( $1 \mathrm{~mA}, 60$ $\mathrm{Hz}, 1 \mathrm{~s}$ ) stimulations to the tail. This is the amount of stress reported to be sufficient to induce the opposite effects of stress on classical eyeblink conditioning (Shors and Servatius, 1997; Shors, 2004). Moreover, it is also important to note that the sex difference in the stress-induced effects on learning is not due to differential activation of pain sensitivity or alterations in the unconditioned response after the stressor (Wood and Shors, 1998; Bangasser and Shors, 2004). In experiment 2, following infusions, unstressed animals were also taken into the context in which stressed animals were exposed to the stressor but were only loosely restrained for $30 \mathrm{~min}$ in the chambers before being returned to their home cages.

Classical conditioning. Twenty-four hours later, rats were returned to the conditioning chamber and exposed to 10 white noise stimuli alone $(250 \mathrm{~ms}, 80 \mathrm{~dB}$, ITI $25 \pm 5 \mathrm{~s}$ ) before the first session. This procedure is used to assess potential effects of the stressor or infusion on sensitized responses to the CS (blinks during first $100 \mathrm{~ms}$ of the CS) (Servatius and Shors, 1994). The rats were then trained with 400 trials (100 trials/d) of paired stimuli using a $80 \mathrm{~dB}, 850 \mathrm{~ms}$ burst of white noise CS overlapping with a $100 \mathrm{~ms}, 0.5 \mathrm{~mA}$ periorbital stimulation of the eyelid (US). These studies were conducted using a delay conditioning procedure in which the CS and the US overlap in time and coterminate. Eyeblinks were assessed by significant changes in the magnitude of the EMG response recorded from the eyelid muscles. Activity that lasted $10 \mathrm{~ms}$ and exceeded $0.3 \mathrm{mV}$ and $4 \mathrm{SD}$ above baseline activity recorded during a $250 \mathrm{~ms}$ pre-CS period was considered indicative of an eyeblink.

Histology. After behavioral testing, rats were given a lethal dose of sodium pentobarbital $(100 \mathrm{mg} / \mathrm{kg})$ and transcardially perfused with $0.9 \%$ saline solution followed by $10 \%$ buffered formalin. Brains were extracted and post-fixed in formalin for at least $24 \mathrm{~h}$. The brains were then cryoprotected in a $30 \%$ sucrose-formalin solution for at least $3 \mathrm{~d}$, after which the brains were frozen and sectioned into $40-\mu \mathrm{m}$-thick coronal sections using a cryostat. Every third section was mounted onto gelled slides and stained with $0.1 \%$ cresyl violet to verify the accuracy of cannula placements. In experiment 2, before brain extraction, a current of $200 \mu \mathrm{A}$ was passed through the microinjector tips, which were inserted into the bilateral cannula, for $20 \mathrm{~s}$ to electrolytically lesion cannula tip sites for verification. These brain slices were then stained with $0.1 \%$ neutral red and potassium ferrocyanide to stain for the electrolytic lesions marking cannula tip placements. A rater, blind to behavioral data, assessed cannula placements. The locations were considered accurate if the tip of the injection cannula, which protruded $0.5 \mathrm{~mm}$ beyond the guide cannula, was within the dorsal boundary of the prelimbic cortex and at least $1 \mathrm{~mm}$ above the ventral boundary of the infralimbic cortex. Placements within the $\mathrm{mPFC}$ were between +3.20 and $+2.70 \mathrm{~mm}$ relative to bregma. A reconstruction of placements that were considered accurate is shown in Figure 1. The site of drug infusion was assessed by track markings of the infusion cannula. Rats were excluded from analysis if placements were not within the $\mathrm{mPFC}$ or if the mPFC was excessively damaged by the cannula or the infusion.

The total number of animals for each group with the appropriate cannula placement are as follows: Experiment 1: males/vehicle/no stress: $n=8$; males/vehicle/stress: $n=8$; males/muscimol/no stress: $n=8$; males/muscimol/stress: $n=7$; females/vehicle/no stress: $n=10$; females/vehicle/stress: $n=8$; females/muscimol/no stress: $n=6$; females/muscimol/stress: $n=8$; experiment 2: males/vehicle/no stress: $n=6$; males/vehicle/stress: $n=5$; males/picrotoxin/no stress: $n=7$; males/picrotoxin/stress: $n=6$; females/vehicle/no stress: $n=7$; females/vehicle/stress: $n=5$; females/ picrotoxin/no stress: $n=6$; females/picrotoxin/stress: $n=7$.

\section{Experiment 3: $m P F C \leftrightarrow B L A$ disconnection}

Subjects. Cycling female Sprague Dawley rats between 90 and $120 \mathrm{~d}$ of age were obtained from a breeding facility at Rutgers University. Rats were housed in groups of 3-4 until surgery. Following surgery, rats were housed alone in standard plastic "shoebox" cages $(44.5 \mathrm{~cm}$ long, $21.59 \mathrm{~cm}$ wide, and $23.32 \mathrm{~cm}$ high). Rats had ad libitum access to rat chow and water and were maintained on a $12 \mathrm{~h}$ light/dark cycle. All experiments were conducted with full compliance to the rules and regulations specified by the PHS Policy on Humane Care and Use of Laboratory Animals and the Guide for the Care and Use of Laboratory Animals.

Vaginal cytology. As in the first experiment, phases of the estrus cycle were monitored via lavage and rats without normal cycles were eliminated from the study. 
Surgery. Female rats were anesthetized with sodium pentobarbital ( $40 \mathrm{mg} / \mathrm{kg}$ for females). After being placed in the stereotaxic instrument, the scalp was cleaned with Betadine, and an incision was made. Excitotoxic lesion sites were infused with NMDA via a $10 \mu \mathrm{l}$ Hamilton syringe attached to a microinfusion pump. For lesions of the medial prefrontal cortex, the syringe tip was aimed at the prelimbic/infralimbic junction (AP: $+3.0 /+2.5 \mathrm{~mm}$; ML: \pm 0.7 $\mathrm{mm}$; DV: $-4.5 \mathrm{~mm}$ from skull), and $0.1 \mu \mathrm{l}$ of $10 \mathrm{mg} / \mathrm{ml}$ NMDA was infused at a rate of 0.1 $\mu \mathrm{l} / \mathrm{min}$. Coordinates for lesions of the basolateral amygdala were as follows: AP: $-2.8 \mathrm{~mm}$; ML: $\pm 4.8 \mathrm{~mm}$; DV: $-8.5 /-8.3 \mathrm{~mm}$ from skull (20 mg/ml NMDA; volume: $0.25 \mu \mathrm{l} / 0.15 \mu \mathrm{l}$; rate: $0.1 \mu \mathrm{l} / \mathrm{min}$ ). All contralateral and ipsilateral lesions (diagrammed in Fig. 2) were counterbalanced and subsequently assessed for possible lateralization effects. Following infusions of excitotoxin, headstages were fitted onto the skull with dental cement and anchored by skull screws. The headstages were attached to four electrodes; two delivered the US of periorbital stimulation and two recorded EMG activity as a measure of blinks. The electrodes were implanted as described in experiment 1.

Stress procedure. At least $7 \mathrm{~d}$ were allowed for recovery time after surgery. Cycling rats in diestrus 2 were placed into the conditioning chamber for an acclimation period. Rats to be stressed were then transferred to a separate room (different from that in which conditioning occurred) into an enclosed soundproof box and underwent brief stress exposure as described in experiment 1.

Classical conditioning. Before either stress exposure or none, the rats were placed into the conditioning boxes for a habituation period in which they acclimated for $1 \mathrm{~h}$ while spontaneous blinks were recorded and then $24 \mathrm{~h}$ later, were returned to the chamber. As in experiment 1 , rats were observed for a sensitization period and then began training with delay eyeblink conditioning.

Histology. After behavioral testing, rats were administered a lethal dose of sodium pentobarbital $(100 \mathrm{mg} / \mathrm{kg})$ and transcardially perfused with $0.9 \%$ saline solution followed by $10 \%$ buffered formalin. Brains were extracted and post-fixed in formalin for at least $24 \mathrm{~h}$. The brains were then cryoprotected in a $30 \%$ sucrose-formalin solution for at least $3 \mathrm{~d}$, after which the brains were frozen and sectioned into $50-\mu \mathrm{m}$-thick coronal sections using a cryostat. Every third section was mounted onto gelled slides and stained with the cresyl violet to verify lesion size. A rater, blind to the behavioral data, assessed lesion placements. Rats were excluded from the study if lesions were misplaced or incomplete. Lesions were identified by the location of the needle track, absence of nerve cell bodies, and gliosis, or the presence of darkly stained astrocytes (Bangasser et al., 2005). The extent of the smallest and largest lesion is presented in Figure 3. The number of remaining animals were as follows: ipsilateral/no stress: $n=10$; ipsilateral/ stress: $n=10$; contralateral/no stress: $n=10$; contralateral/stress: $n=10$.

\section{Results}

Experiments 1 and 2: mPFC activity in males versus females In the first experiment, the $\mathrm{mPFC}$ was inactivated during the stressor in males and females. All animals were trained with delay

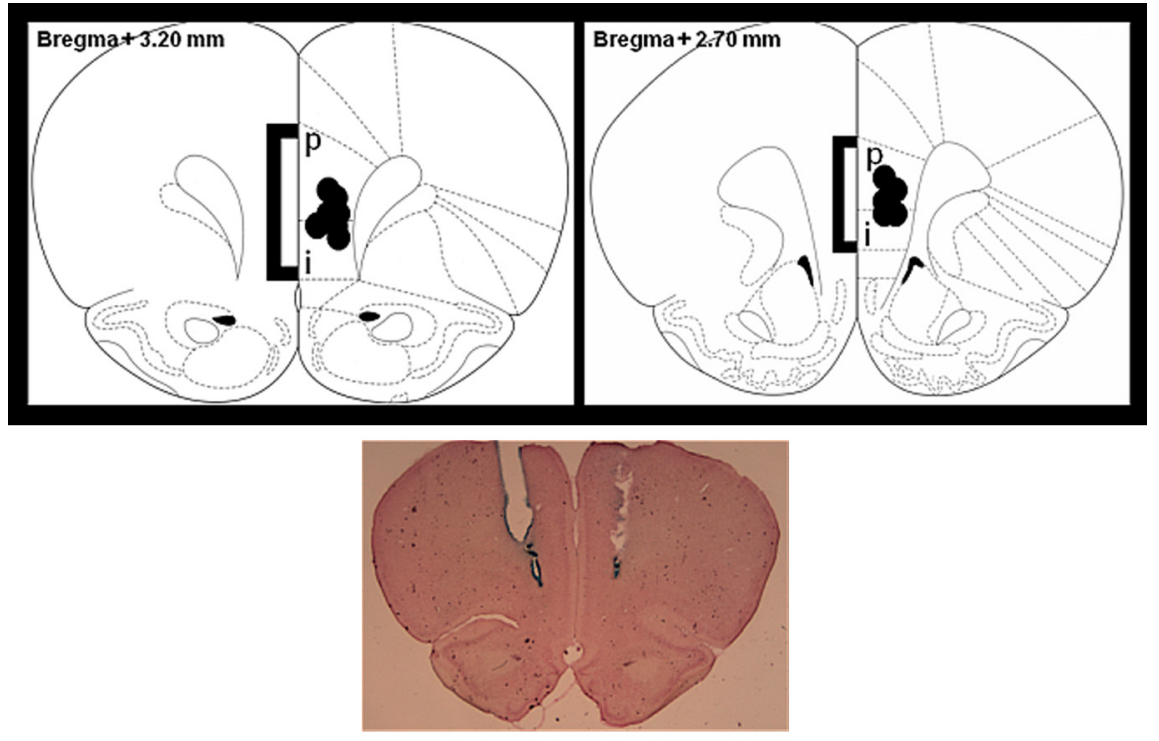

Figure 1. Histology of mPFC cannulation. Cannula tip placements within the mPFC were between +3.20 and $+2.70 \mathrm{~mm}$ relative to bregma. Animals were included if tips of the injection cannula were within the dorsal boundary of the prelimbic cortex with cannula tip placements marked by Prussian blue is also included. p, Prelimbic region of the medial prefrontal cortex; I infralimbic region of the medial prefrontal cortex.

mPFC-BLA disconnection procedure. Animals with contralateral excitotoxic lesions received a unilateral lesion to the $\mathrm{mPFC}$ and a unilateral lesion to the BLA in opposite hemispheres. The contralateral lesion disrupted communication between the MPFC and BLA in both hemispheres. Animals with ipsilateral lesions received a unilateral lesion of the MPFC and a unilateral lesion these rats. If the concurrent activation of both the $\mathrm{MPFC}$ and BLA is necessary for the learning deficit after stress, then animals with contralateral lesions should learn well even though they were exposed to the stressor.

conditioning $24 \mathrm{~h}$ later. Anticipatory conditioned responses (CRs) before the US were counted and averaged across blocks of 100 trials (Fig. 4). Males were analyzed separately from females. Four groups of males were trained: one group whose $\mathrm{mPFC}$ was inactivated during the stressor, one with MPFC inactivation and no stressor exposure, another stressed and injected with aCSF, and a vehicle group that was not stressed. The independent measures were stress versus no stress and inactivation with muscimol versus aCSF vehicle infusion.

To assess acquisition of the $\mathrm{CR}$ across trials of training in males, a $2 \times 2$ repeated-measures ANOVA across the four sessions of trials was conducted on each group. The analysis revealed an effect of session as the number of CRs increased as training progressed $\left(F_{(3,81)}=19.99 ; p<0.01\right)$. There was an effect of stress 
A
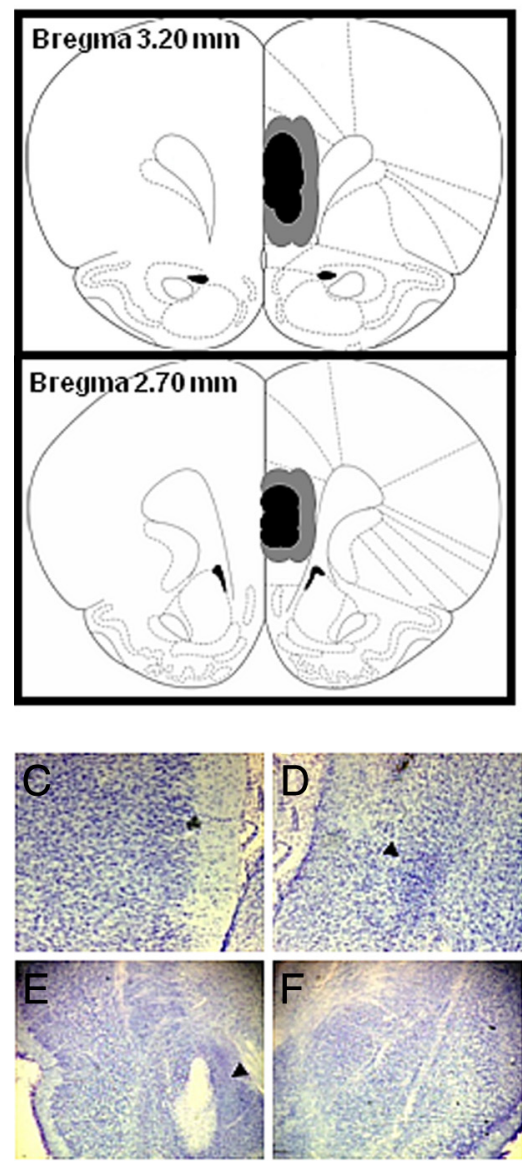

B

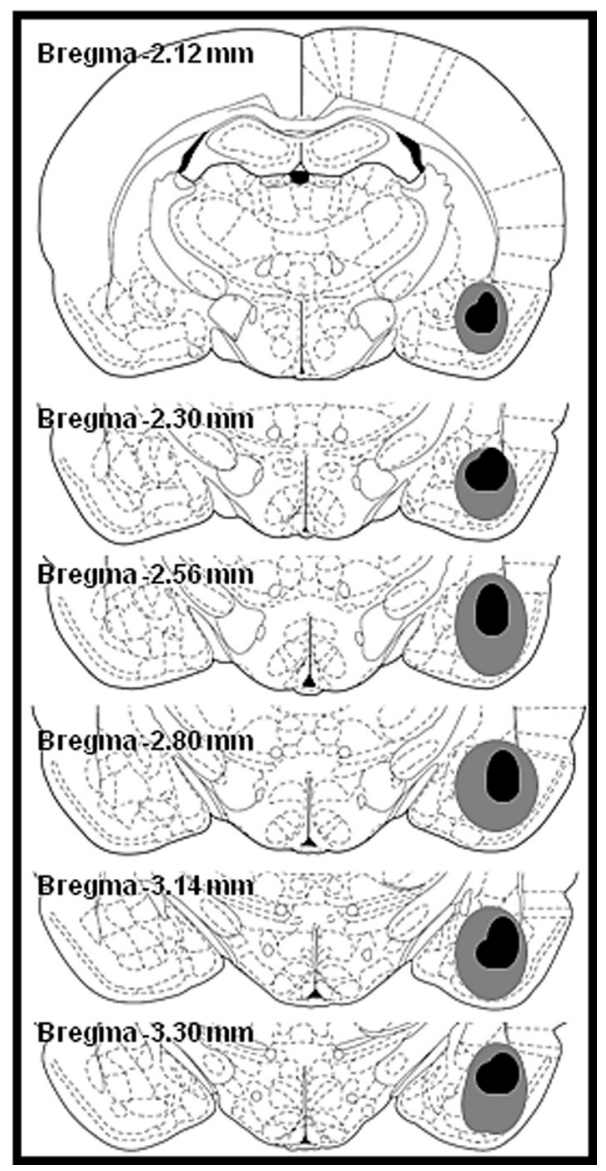

Figure 3. Histology of mPFC-BLA disconnection. $\boldsymbol{A}, \mathrm{mPFC}$ lesions. $\boldsymbol{B}, \mathrm{BLA}$ lesions. Largest lesions (in gray) and smallest lesions (in black) of rats included are depicted here. The unilateral images are representative of lesions in both hemispheres. Brain sections of the $\mathrm{MPFC}$ and BLA were stained with $0.1 \%$ cresyl violet to verify sites of excitotoxic damage (marked by arrowheads). Note the darkly stained astrocytes and absence of cell bodies in lesioned tissue $(4 \times)$. C, Intact mPFC. D, Lesioned mPFC. $\boldsymbol{E}$, Lesioned BLA. $\boldsymbol{F}$, Intact BLA.

$\left(F_{(1,27)}=19.67 ; p<0.01\right)$ but no effect of muscimol $\left(F_{(1,27)}=\right.$ $0.15 ; p>0.05)$. Furthermore, there was no interaction between muscimol treatment and stress $\left(F_{(1,27)}=0.35 ; p>0.05\right)$. Males that were exposed to the stressor emitted more CRs than those that were not exposed to the stressor regardless of drug treatment $(p<0.01)$ (Fig. 4A). The increase in responding occurred regardless of whether or not the MPFC was inactivated with muscimol during the stressor. Thus, muscimol infusion before stress exposure did not abolish the subsequent facilitation of eyeblink conditioning elicited by stress in males.

To evaluate early acquisition, the first five blocks of 20 trials were analyzed with a $2 \times 2$ repeated-measures ANOVA. Again, there was no effect of muscimol $\left(F_{(1,27)}=0.77 ; p>0.05\right)$, nor was there an interaction between muscimol and stress $\left(F_{(1,27)}\right.$ $=0.29 ; p>0.05)$. However, there was a main effect of blocks of trials $\left(F_{(4,108)}=12.90 ; p<0.01\right)$ as males increased their conditioned responding across trial blocks. There was also a main effect of stress $\left(F_{(1,27)}=6.70 ; p<0.05\right)$. Thus, there was no effect of muscimol inactivation but an effect of stress on both early acquisition and later conditioned responding in males.

Four groups of females were also trained: one group whose $\mathrm{mPFC}$ was inactivated during the stressor, one with $\mathrm{mPFC}$ inactivation without exposure to the stressor, another stressed and injected with aCSF, and an unstressed vehicle group (Fig. 4B). To

assess acquisition across training in females, a $2 \times 2$ repeated-measures ANOVA across the four sessions of trials was conducted on each group. The analysis revealed an effect of session, as conditioned responding increased across training days $\left(F_{(3,81)}=\right.$ 37.28; $p<0.01)$. However, stressed females with vehicle infusions did not express such an increase $\left(F_{(3,21)}=2.96 ; p>\right.$ $0.05)$ contrary to the remaining 3 groups that did increase. Using the percentage of CRs across trials of training as the dependent measure, there was a main effect of muscimol $\left(F_{(1,27)}=8.06 ; p<0.01\right)$ and stress $\left(F_{(1,27)}=7.63 ; p<0.05\right)$. More critically, there was an interaction between the muscimol inactivation and stress exposure $\left(F_{(1,27)}=9.12 ; p<0.01\right)$. A Newman-Keuls post hoc test confirmed that the females that were injected with vehicle in the mPFC before the stressor emitted fewer responses than those that were not stressed and received vehicle $(p<0.01)$. Furthermore, females that were injected with muscimol and stressed emitted more CRs than those that were injected with vehicle and stressed $(p<0.01)$. Also, females that were only injected with muscimol emitted similar numbers of CRs when compared with females that were injected with saline $24 \mathrm{~h}$ before training $(p<0.01)$. Thus, muscimol alone did not alter responding $24 \mathrm{~h}$ later. This result indicates that the stress-induced impairment was prevented in muscimol-infused females because the mPFC was inactivated and not because muscimol alone was altering or enhancing the response. Collectively, these data indicate that neuronal activity within the $\mathrm{mPFC}$ during a stressor is necessary to impair performance of the CR in females.

To assess the effects of stress or muscimol infusion on early acquisition, a $2 \times 2$ repeated-measures ANOVA was conducted on five 20 trial blocks for the first 100 trials. There was no effect of muscimol $\left(F_{(1,27)}=2.29 ; p>0.05\right)$ or stress $\left(F_{(1,27)}=\right.$ $1.24 ; p>0.05)$ and no drug $\times$ stress interaction $\left(F_{(1,27)}=1.84\right.$; $p>0.05)$. However, there was an effect of blocks $\left(F_{(4,108)}=11.31\right.$; $p<0.01)$, as the animals learned and increased responding as blocks of trials proceeded. However, a one-way repeatedmeasures ANOVA revealed again that this increase in responding across blocks as the animals learned did not occur in stressed females with vehicle infusions $\left(F_{(4,28)}=1.34 ; p>0.05\right)$, which persisted throughout the remaining three sessions. Therefore, stress or drug infusion did not differentially influence early responding but rather altered performance in females later in the training sessions.

In experiment 2, the $\mathrm{MPFC}$ was activated with GABA-A receptor antagonist picrotoxin during and in the absence of the stressor in males and females. As in the first experiment, all animals were trained with delay conditioning $24 \mathrm{~h}$ later, and the same CR measurements were assessed (Fig. 5). Again, males were analyzed separately from females. Four groups of males were trained: one group whose $\mathrm{MPFC}$ was activated during the stressor, one with $\mathrm{mPFC}$ ac- 
A

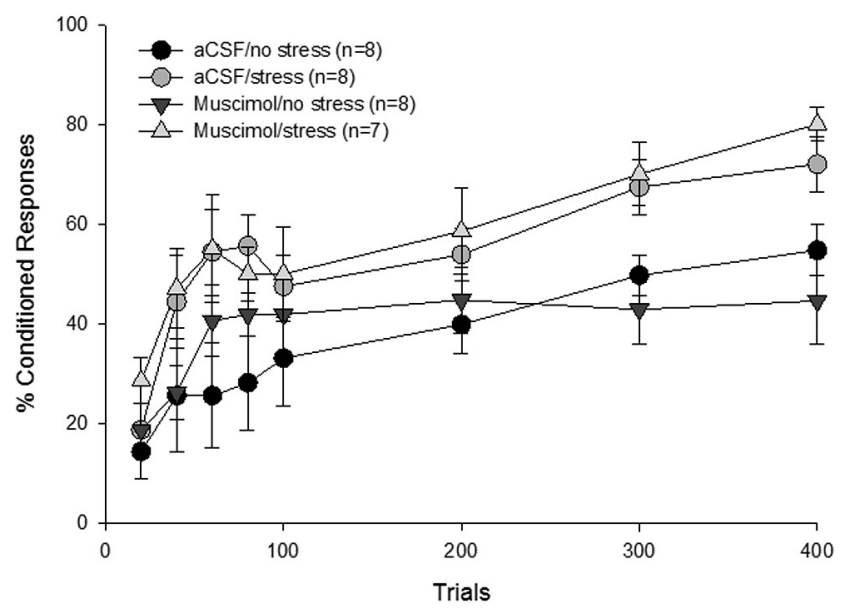

B

Females

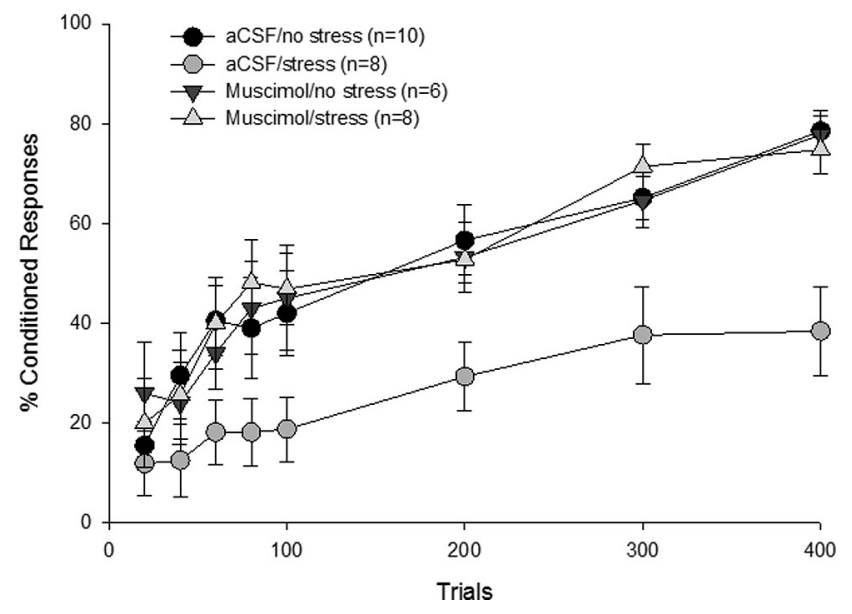

Figure 4. mPFC inactivation. The mPFC was inactivated with muscimol or infused with aCSF during the stressor. One day later, animals were trained with delay conditioning. $A$, Males treated with muscimol and stressed emitted more CRs than those treated with either vehicle or muscimol and not stressed. They performed similarly to those that were stressed and infused with aCSF. Thus, inactivating the mPFC did not prevent the stress-induced facilitation of learning in males. $\boldsymbol{B}$, Stressed females infused with vehicle expressed fewer CRs than their unstressed counterparts. However, when females were infused with muscimol during the stressor, they increased responding and performed similarly to those that were unstressed. Thus, in contrast to males, $\mathrm{mPFC}$ inactivation in females eliminated the modified behavioral response after stress.

tivation without tail shock stressor exposure, another stressed and injected with saline, and a saline group that was not tail shock stressed. The independent measures were stress versus no stress and activation with picrotoxin versus saline vehicle infusion.

To assess acquisition of the CR across trials of training in males, a $2 \times 2$ repeated-measures ANOVA across the four sessions of trials was conducted on each group. All four groups increased the number of CRs as training progressed $\left(F_{(3,60)}=\right.$ $7.18 ; p<0.01)$. There was no effect of picrotoxin $\left(F_{(1,20)}=0.23\right.$; $p>0.05)$ and no interaction between picrotoxin treatment and stress $\left(F_{(1,20)}=0.21 ; p>0.05\right)$. However, there was an effect of stress $\left(F_{(1,20)}=9.62 ; p<0.01\right)$. Males injected with saline before the stressor emitted more CRs than males that were unstressed and injected with saline $(p<0.01)$. Furthermore, unstressed males treated with picrotoxin performed similarly to those treated with the vehicle $(p>0.05)$. Most importantly, males that were exposed to the stressor emitted more CRs than those that
A

Males

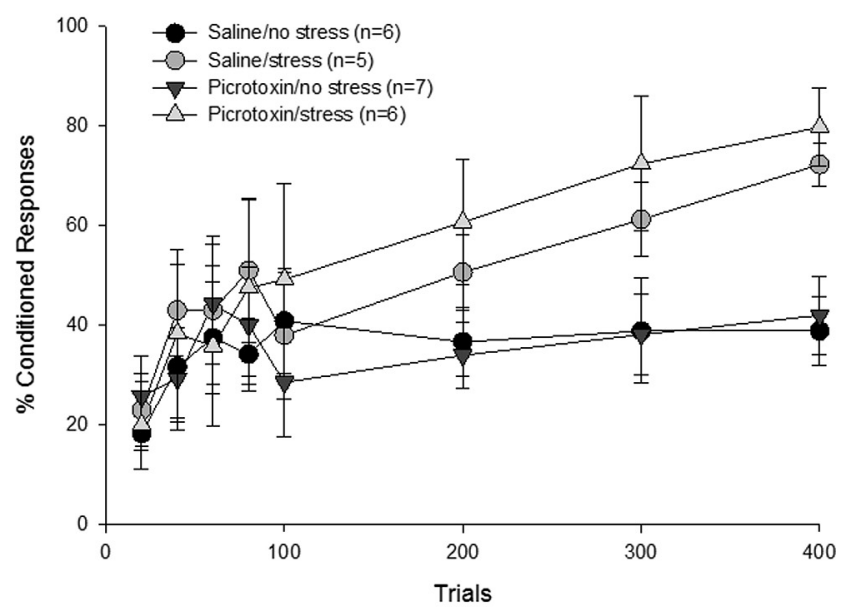

B

Females

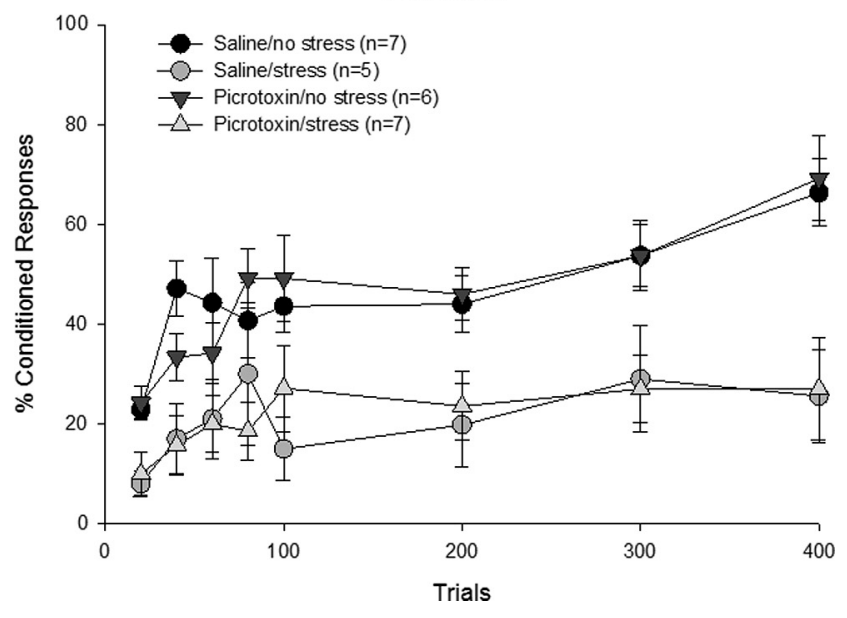

Figure 5. $\mathrm{mPFC}$ activation. The $\mathrm{mPFC}$ was infused with picrotoxin or saline during the stressor. One day later, animals were trained with delay conditioning. A, Picrotoxin-treated stressed males emitted more CRs than the saline-treated unstressed males but performed similarly to those that were stressed and infused with saline. Thus, activating the mPFC did not alter the stress-induced facilitation of learning or performance in the absence of stress in males. $\boldsymbol{B}$, Stressed females infused with saline expressed fewer CRs than their unstressed counterparts. Picrotoxin-infused stressed females performed similarly to the saline-treated stressed females, emitting fewer CRs than picrotoxin-treated unstressed animals. Thus, mPFC activation in females had no effect on decremented conditioned responding following stress exposure or performance in the absence of stress.

were not exposed to the stressor $(p<0.01)$ regardless of the presence of picrotoxin (Fig. $5 A$ ). The increase in responding occurred regardless of whether or not the MPFC was activated with picrotoxin during the stressor. Thus, picrotoxin infusion before stress exposure did not alter the subsequent facilitation of eyeblink conditioning elicited by stress in males.

The first five blocks of 20 trials were analyzed with a $2 \times 2$ repeated-measures ANOVA. There was no effect of picrotoxin $\left(F_{(1,20)}=0.00 ; p>0.05\right)$, stress $\left(F_{(1,20)}=0.27 ; p>0.05\right)$, with no interaction between picrotoxin treatment and stress $\left(F_{(1,20)}=\right.$ $0.01 ; p>0.05)$. However, there was a main effect of blocks of trials $\left(F_{(4,80)}=9.04 ; p<0.01\right)$, as males increased their conditioned responding across trial blocks. Thus, there was no effect of picrotoxin treatment or stress on early learning with changes emerging later in conditioning.

Four groups of females were also trained: one group whose $\mathrm{mPFC}$ was activated during the stressor, one with $\mathrm{mPFC}$ activation 
without exposure to the stressor, another stressed and injected with saline, and an unstressed saline group (Fig. 5B). To assess acquisition across training in females, a $2 \times 2$ repeated-measures ANOVA across the four sessions of trials was conducted on each group. The analysis revealed an effect of session, as conditioned responding increased across training days $\left(F_{(3,63)}=8.19 ; p<0.01\right)$. However, a one-way repeated-measures ANOVA revealed that stressed females did not show an increase in responding across sessions with saline $\left(F_{(3,12)}=0.47 ; p>0.05\right)$ or even picrotoxin infusions $\left(F_{(3,18)}=0.94 ; p>0.05\right)$, whereas unstressed animals did. There was no effect of picrotoxin $\left(F_{(1,21)}=0.02 ; p>0.05\right)$ and no interaction between picrotoxin treatment and stress $\left(F_{(1,21)}=\right.$ $0.00 ; p>0.05)$. Using the percentage of CRs across trials of training as the dependent measure, there was a main effect of stress $\left(F_{(1,21)}=27.77 ; p<0.01\right)$. Females that were injected with a vehicle in the mPFC before the stressor emitted fewer responses than those that were not stressed and received vehicle or picrotoxin infusions $(p<0.01)$. Moreover, females that were stressed while their mPFC was activated also did not learn well, emitting fewer CRs than those that were unstressed and microinjected with the vehicle or picrotoxin $(p<0.01)$ and performing similarly to the vehicle-treated stressed females $(p>0.05)$. Together, these data suggest that stress impairs subsequent learning via neuronal activity within the mPFC during the stressor in females, but activation of this brain region alone may not be sufficient.

To assess the effects of stress or picrotoxin infusion on early acquisition, a $2 \times 2$ repeated-measures ANOVA was run on five 20 trial blocks for the first 100 trials. There was no effect of $\operatorname{drug}\left(F_{(1,21)}=0.02 ; p>0.05\right)$ and no drug $\times$ stress interaction $\left(F_{(1,21)}=0.03 ; p>0.05\right)$. However, there was an effect of stress $\left(F_{(1,21)}=14.85 ; p<0.01\right)$ and blocks $\left(F_{(4,84)}=10.57 ; p<\right.$ $0.01)$, as the animals learned and increased responding as blocks of trials proceeded. However, a one-way repeated-measures ANOVA revealed that this increase in responding across blocks did not occur in stressed females with vehicle $\left(F_{(4,16)}=2.54 ; p>\right.$ $0.05)$ as well as picrotoxin infusions $\left(F_{(4,24)}=2.35 ; p>0.05\right)$. Thus, there was no effect of picrotoxin but an effect of stress on early acquisition as well as later conditioned responding.

\section{Experiment 3: Communication between the mPFC and the amygdala in females}

Experiment 3 assessed whether communication between the mPFC and BLA was necessary to impair learning in females after acute stress exposure. To do so, females were stressed either with ipsilateral or contralateral lesions to the mPFC and BLA. The behavioral results are presented in Figure 6. A $2 \times 2$ repeatedmeasures ANOVA across the four sessions of 100 trials of training revealed an effect of session $\left(F_{(3,108)}=33.00, p<0.01\right)$, indicating that animals increased their conditioned responding as training progressed. However, a one-way repeated-measures ANOVA revealed that this increase in responding across sessions indicative of learning did not occur in stressed females with ipsilateral lesions $\left(F_{(3,27)}=0.49 ; p>0.05\right)$ contrary to the remaining 3 groups that learned. The main effects of lesion $\left(F_{(1,36)}=6.33, p<\right.$ $0.05)$ and stress $\left(F_{(1,36)}=8.81, p<0.01\right)$ were also significant. Furthermore, there was a three-way interaction (lesion $\times$ stress $\times$ session $)\left(F_{(3,108)}=3.26, p<0.05\right)$. A Newman-Keuls post hoc analysis confirmed that females that were stressed with lesions on the same side of the brain responded with fewer CRs than those that were not stressed with the same type of lesions $(p<0.01)$. Furthermore, females in this group (stressed with ipsilateral lesions) also emitted fewer CRs than those in the other three groups $(p<0.01)$. However, those that were stressed with lesions on

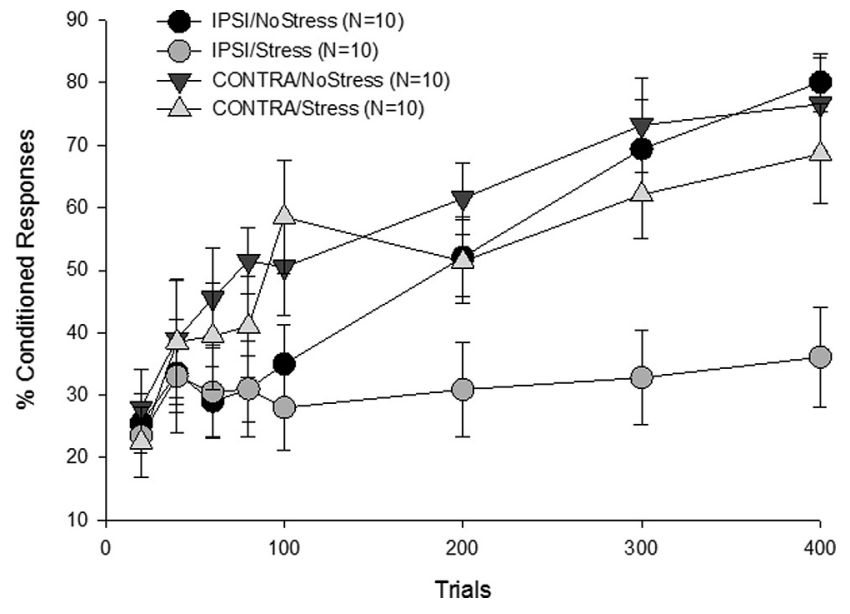

Figure 6. mPFC-BLA disconnection. Acute stressful experience disrupted learning in females with ipsilateral mPFC-BLA lesions. In contrast, conditioned responding of animals with contralateral lesions was not impaired by stress and was similar to the performance of the unstressed females with both types of lesions. Thus, communication between the MPFC and BLA is necessary to impair learning after a stressful event in females.

opposite sides of the brain performed no differently than their unstressed counterparts $(p>0.05)$. Importantly, the lesions did not alter performance itself. Females that were not stressed but had lesions on the same side performed similarly to those that were not stressed and had lesions on the opposite sides $(p>0.05)$.

To assess learning in the early trials of training, a repeatedmeasures ANOVA was conducted on the first 100 trials, which are presented in Figure 5 in blocks of 20 trials. There was no main effect of lesion $\left(F_{(1,36)}=3.30, p>0.05\right)$ nor stress $\left(F_{(1,36)}=0.13, p>\right.$ $0.05)$. However, there was an effect of blocks of trials $\left(F_{(4,144)}=\right.$ $10.13, p<0.01)$. A one-way repeated-measures ANOVA revealed that stressed females with ipsilateral lesions did not alter their responding across blocks $\left(F_{(4,36)}=0.78, p>0.05\right)$. Therefore, these females did not learn within the four sessions of training. In summary, the effects of lesion and stress on learning were not apparent during the first session of training but rather emerged during training on subsequent days.

Rats were considered to have learned the response if they emitted at least $60 \%$ CRs during two consecutive sessions of training. Using this criterion, we determined that all of the groups learned except the group of stressed females with ipsilateral lesions of the mPFC and the BLA (Fig. 7). Thus, stressed females whose $\mathrm{mPFC}$ and amygdala were still in communication did not learn, whereas those that had disrupted communication on each side learned the CR as well as those that were not exposed to the stressor. These data indicate that communication between the mPFC and BLA is necessary to impair associative eyeblink conditioning in females after an acute stressful event.

\section{Discussion}

Exposure to an acute stressful event enhances classical eyeblink conditioning in males but profoundly disrupts this type of learning in females (Wood and Shors, 1998). Once stressed, most females emit drastically fewer conditioned responses, a learning deficit that has been shown to persist even after hundreds of trials of training (Shors et al., 1998; Wood and Shors, 1998; Wood et al., 2001; Leuner and Shors, 2006). Here we report that the mPFC is critically involved during the stressor to induce the impairment. This was determined by inactivating the brain region with muscimol during the stressful event and training animals $24 \mathrm{~h}$ later in the absence of the drug. When the mPFC was functionally inac- 


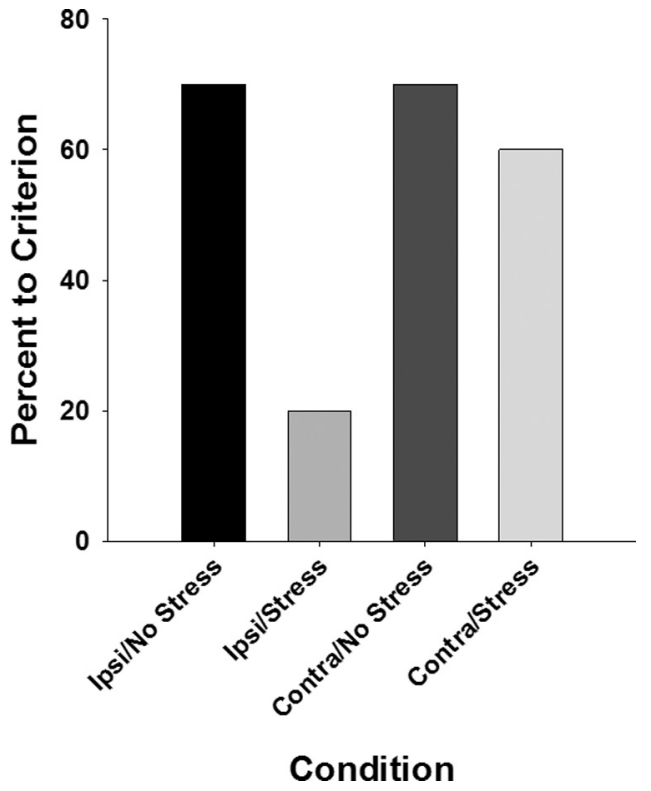

Figure 7. The percentage of female rats in each lesion and stress condition that met learning criterion. Animals that learned emitted at least $60 \%$ CRs in at least two (of the four) consecutive sessions of 100 trials.

tivated, stressed females learned well. Given the time course, we conclude that the mPFC is critically involved during the stressful event to impair learning in the future. Interestingly enough, inactivation of the mPFC during the stressful event did not prevent the enhanced conditioning in males. Thus, neural activity within the mPFC is necessary to disrupt learning in females but not necessary to enhance performance in males after stress. These results are novel because they indicate that activity within the $\mathrm{mPFC}$ during a stressful event is necessary to impair future learning, specifically in females.

It was somewhat surprising that activity within the mPFC was not necessary to enhance learning in males. Most experiments are conducted exclusively in males and many indicate that the mPFC is involved in processes of associative learning, including eyeblink conditioning (Goldman-Rakic, 1995; Kronforst-Collins and Disterhoft, 1998; Fuster, 2001; Takehara et al., 2002). Furthermore, the mPFC is active during stress (Cerqueira et al., 2008), densely populated with glucocorticoid receptors (Lupien and Lepage, 2001), and plays a role in the hypothalamo-pituitary-adrenal response to stress (Diorio et al., 1993; Figueiredo et al., 2003; Radley et al., 2006). In humans, stress-related disorders have been associated with differences in both the structure and function of the mPFC (Bremner et al., 1999; Drevets, 2000; Rajkowska, 2000). Similarly, stress exposure induces dendritic remodeling within the mPFC (Brown et al., 2005; Garrett and Wellman, 2009; Shansky and Morrison, 2009). Others find that the mPFC modulates the effects of controllability on processes of learning. Specifically, inactivation of the mPFC during the stressor prevented the protective effects of "controllability" on helplessness behavior. These animals also expressed more fear during conditioning, even though they could control the stress (Amat et al., 2005). Controllability does eliminate the effects of stress on eyeblink conditioning in both males and females (Leuner et al., 2004), although the role of the mPFC in this phenomenon has not been examined.

To further explore the idea that stress engages this specific brain region to modify learning after stress, the mPFC was acti- vated via infusions of picrotoxin in the presence and absence of the stressor. If the stress effect on learning occurs solely through activation of the mPFC, then its activation in absence of the stressor should mimic the effect of the stressor and thereby impair learning in females and/or facilitate learning in males. In experiment 2, we report that activation of the $\mathrm{mPFC}$ with picrotoxin did not alter learning $24 \mathrm{~h}$ later in males or females. Thus, activation of the mPFC alone is not sufficient to induce the stress effects on learning. The data demonstrate that activation of the mPFC did not alter or itself induce the stress effect on learning in either sex. Because neither inactivation nor activation of the $\mathrm{MPFC}$ during the stressor altered learning in males, the $\mathrm{MPFC}$ is not part of the critical circuitry necessary to enhance conditioning. In contrast, excitatory neuronal activity within the MPFC during the stressor is necessary in females to modulate learning, but excitation alone is not sufficient.

The data from the first two experiments indicate that the $\mathrm{mPFC}$ is critical for the stress effect on learning in females but not in males. Because mPFC excitation alone did not impair learning in females, we hypothesized that the $\mathrm{mPFC}$ is interacting with additional brain regions to impair learning. We investigated the BLA, because it is a critical brain structure in the modulation of learning after stress (Waddell et al., 2008). In the third experiment, we determined that the mPFC communicates with the BLA to impair learning after stress. Females with unilateral lesions to each structure on opposite sides of the brain (i.e. those in which both connections were disrupted) were able to learn well after the stressor. In contrast, those with lesions to the same hemisphere (only one connection disrupted) did not learn well after stress. It is presumed that the learning deficit was maintained by the one intact connection between the $\mathrm{MPFC}$ and BLA. If anything, most lesions impair processes of learning. Conceptually, it is interesting that the complete absence of an anatomical connection can facilitate the learning process.

There are many reciprocal anatomical and physiological interactions between the mPFC and the amygdala (Krettek and Price, 1977; Porrino et al., 1981; Quirk et al., 2003; Hoover and Vertes, 2007). Notably, there are direct projections from the mPFC to the amygdala and extended amygdala (McDonald, 1991; McDonald et al., 1999). Most studies indicate that the mPFC suppresses amygdalar activity, even though most corticoamygdalar projections are excitatory (Rosenkranz and Grace, 2001; Sotres-Bayon et al., 2004). This may occur via excitation of GABAergic BLA interneurons that decrease excitatory input to the central nucleus (Grace and Rosenkranz, 2002). There are also projections from the basolateral amygdala to the frontal cortex (Kita and Kitai, 1990), especially to cells in mPFC layers II and V (Bacon et al., 1996). Stimulation of the BLA modifies neuronal responses in the $\mathrm{mPFC}$, and based on latency, some connections are monosynaptic while others are polysynaptic (PérezJaranay and Vives, 1991). That said, projection neurons from the BLA to the $\mathrm{mPFC}$ are immunoreactive for glutamate and/or aspartate, indicating direct monosynaptic excitatory input to the mPFC from the BLA (McDonald et al., 1989).

Communication between the mPFC and the amgydala does interact with processes related to the stress response and to learning. The mPFC can suppress activity within the BLA when activated first (Rosenkranz and Grace, 2001; Sotres-Bayon et al., 2004; Likhtik et al., 2005). mPFC stimulation can reduce physiological responses in the central nucleus, which may thereby influence activity in the BLA via inhibitory intercalated cells (Paré, 2003; Quirk et al., 2003). We would suggest that the deficit in learning examined here is mediated by activity within the $\mathrm{MPFC}$, 
which then modulates the expression of fear during aversive learning via output from the amygdala. Indeed, many studies report inhibitory control of the amygdala by the mPFC during emotional learning, such as during extinction (Morgan et al., 1993; Morgan and LeDoux, 1995; Quirk et al., 2000; SotresBayon et al., 2004). Alternatively, the amygdala may modulate activity in the mPFC to impair learning. Acute stress exposure prevents the induction of LTP in vivo within the $\mathrm{MPFC}$ in response to stimulation of the BLA-mPFC pathway (Maroun and Richter-Levin, 2003). Others found that BLA stimulation modulates neuronal activity in the mPFC (Pérez-Jaranay and Vives, 1991). Furthermore, fear conditioning, which relies on the amygdala, can inhibit activity of prefrontal cortical neurons (Garcia et al., 1999), again pointing to amygdalar regulation of the mPFC. Alternatively, it could be that concurrent activity within the BLA and the $\mathrm{mPFC}$ is necessary to impair learning after stress. Both structures can modulate performance of the conditioned eyeblink response (Powell et al., 1996; Kronforst-Collins and Disterhoft, 1998; Lee and Kim, 2004). They project not just to each other but to brain structures involved in the limbichypothalamic-pituitary-adrenal stress circuit (López et al., 1999). Previously, we found that the hippocampus is involved in these effects of stress on learning (Bangasser et al., 2007). Thus, activity within the mPFC may communicate with the amygdala by way of the hippocampus.

The vast majority of studies about the MPFC and BLA have been conducted exclusively in males, but a few studies do report sex differences. In ovariectomized females, stress and estrogen together induce dendritic arborization in neurons that project from the MPFC to the BLA when compared with the same measures in males (Shansky and Morrison, 2009). The learning deficit after stress in females depends on the presence of estrogen (Wood and Shors, 1998), and thus it seems likely that estrogen is acting within one and/or the other structure to modulate learning. Regardless of the exact mechanism, the present data indicate that the mPFC and the amygdala interact with each other to impair associative learning, specifically in females. Minimally, they indicate that males and females are using different brain regions and circuits to modify learning after stress. More generally, they may provide clues as to why women are so much more vulnerable than men are to stress-related mental illness, such as post-traumatic stress disorder and depression.

\section{References}

Amat J, Baratta MV, Paul E, Bland ST, Watkins LR, Maier SF (2005) Medial prefrontal cortex determines how stressor controllability affects behavior and dorsal raphe nucleus. Nat Neurosci 8:365-371.

Bacon SJ, Headlam AJ, Gabbott PL, Smith AD (1996) Amygdala input to medial prefrontal cortex (mPFC) in the rat: a light and electron microscope study. Brain Res 720:211-219.

Bangasser DA, Shors TJ (2004) Acute stress impairs trace eyeblink conditioning in females without altering the unconditioned response. Neurobiol Learn Mem 82:57-60.

Bangasser DA, Shors TJ (2007) The hippocampus is necessary for enhancements and impairments of learning following stress. Nat Neurosci 10:1401-1403.

Bangasser DA, Shors TJ (2008) The bed nucleus of the stria terminalis modulates learning after stress in masculinized but not cycling females. J Neurosci 28:6383-6387.

Bangasser DA, Santollo J, Shors TJ (2005) The bed nucleus of the stria terminalis is involved in the persistent increase in associative learning after stress. Behav Neurosci 119:1459-1466.

Bremner JD, Staib LH, Kaloupek D, Southwick SM, Soufer R, Charney DS (1999) Neural correlates of exposure to traumatic pictures and sound in Vietnam combat veterans with and without posttraumatic stress disorder: a positron emission tomography study. Biol Psychiatry 45:806-816.
Brown SM, Henning S, Wellman CL (2005) Mild, short-term stress alters dendritic morphology in rat medial prefrontal cortex. Cereb Cortex 15:1714-1722.

Carter-Snell C, Hegadoren K (2003) Stress disorders and gender: implications for theory and research. Can J Nurs Res 35:34-55.

Cerqueira JJ, Almeida OF, Sousa N (2008) The stressed prefrontal cortex. Left? Right! Brain Behav Immun 22:630-638.

Diorio D, Viau V, Meaney MJ (1993) The role of the medial prefrontal cortex (cingulate gyrus) in the regulation of hypothalamic-pituitaryadrenal responses to stress. J Neurosci 13:3839-3847.

Drevets WC (2000) Neuroimaging studies of mood disorders. Biol Psychiatry 48:813-829.

Figueiredo HF, Bruestle A, Bodie B, Dolgas CM, Herman JP (2003) The medial prefrontal cortex differentially regulates stress-induced c-fos expression in the forebrain depending on type of stressor. Eur J Neurosci 18:2357-2364.

Foa EB, Street GP (2001) Women and traumatic events. J Clin Psychiatry 62 [Suppl 17]:29-34.

Fuster JM (2001) The prefrontal cortex-an update: time is of the essence. Neuron 30:319-333.

Garcia R, Vouimba RM, Baudry M, Thompson RF (1999) The amygdala modulates prefrontal cortex activity relative to conditioned fear. Nature 402:294-296.

Garrett JE, Wellman CL (2009) Chronic stress effects on dendritic morphology in medial prefrontal cortex: sex differences and estrogen dependence. Neuroscience 162:195-207.

Gerrits M, Bakker PL, Koch T, Ter Horst GJ (2006) Stress-induced sensitization of the limbic system in ovariectomized rats is partly restored by cyclic 17 $\beta$-estradiol administration. Eur J Neurosci 23:1747-1756.

Goldman-Rakic PS (1995) Cellular basis of working memory. Neuron 14: 477-485.

Grace AA, Rosenkranz JA (2002) Regulation of conditioned responses of basolateral amygdala neurons. Physiol Behav 77:489-493.

Heidbreder CA, Groenewegen HJ (2003) The medial prefrontal cortex in the rat: evidence for a dorso-ventral distinction based upon functional and anatomical characteristics. Neurosci Biobehav Rev 27:555-579.

Hoover WB, Vertes RP (2007) Anatomical analysis of afferent projections to the medial prefrontal cortex in rat. Brain Struct Funct 212:149-179.

Kessler RC, Sonnega A, Bromet E, Hughes M, Nelson CB (1995) Posttraumatic stress disorder in the National Comorbidity Survey. Arch Gen Psychiatry 52:1048-1060.

Kita H, Kitai ST (1990) Amygdaloid projections to the frontal cortex and the striatum in the rat. J Comp Neurol 298:40-49.

Krettek JE, Price JL (1977) Projections from the amygdaloid complex to the cerebral cortex and thalamus in the rat and cat. J Comp Neurol 172:687-722.

Kronforst-Collins MA, Disterhoft JF (1998) Lesions of the caudal area of rabbit medial prefrontal cortex impair trace eyeblink conditioning. Neurobiol Learn Mem 69:147-162.

Lee T, Kim JJ (2004) Differential effects of cerebellar, amygdalar and hippocampal lesions on classical eyeblink conditioning in rats. J Neurosci 24:3242-3250.

Leuner B, Shors TJ (2006) Learning during motherhood: a resistance to stress. Horm Behav 50:38-51.

Leuner B, Mendolia-Loffredo S, Shors TJ (2004) Males and females respond differently to controllability and antidepressant treatment. Biol Psychiatry 56:964-970.

Likhtik E, Pelletier JG, Paz R, Paré D (2005) Prefrontal control of the amygdala. J Neurosci 25:7429-7437.

López JF, Akil H, Watson SJ (1999) Neural circuits mediating stress. Biol Psychiatry 46:1461-1471.

Luine V (2002) Sex differences in chronic stress effects on memory in rats. Stress 5:205-216.

Lupien SJ, Lepage M (2001) Stress, memory, and the hippocampus: can't live with it, can't live without it. Behav Brain Res 127:137-158.

Maroun M, Richter-Levin G (2003) Exposure to acute stress blocks the induction of long-term potentiation of the amygdala-prefrontal cortex pathway in vivo. J Neurosci 23:4406-4409.

McDonald AJ (1991) Organization of amygdaloid projections to the prefrontal cortex and associated striatum in the rat. Neuroscience 44:1-14.

McDonald AJ, Beitz AJ, Larson AA, Kuriyama R, Sellitto C, Madl JE (1989) Co-localisation of glutamate and tubulin in putative excitatory neurons of 
the hippocampus and amygdala: an immunohistochemical study using monoclonal antibodies. Neuroscience 30:405-421.

McDonald AJ, Shammah-Lagnado SJ, Shi C, Davis M (1999) Cortical afferents to the extended amygdala. Ann N Y Acad Sci 877:309-338.

Morgan MA, LeDoux JE (1995) Differential contribution of dorsal and ventral medial prefrontal cortex to the acquisition and extinction of conditioned fear in rats. Behav Neurosci 109:681-688.

Morgan MA, Romanski LM, LeDoux JE (1993) Extinction of emotional learning: contribution of medial prefrontal cortex. Neurosci Lett 163:109-113.

Paré D (2003) Role of the basolateral amygdala in memory consolidation. Prog Neurobiol 70:409-420.

Pérez-Jaranay JM, Vives F (1991) Electrophysiological study of the response of medial prefrontal cortex neurons to stimulation of the basolateral nucleus of the amygdala in the rat. Brain Res 564:97-101.

Porrino LJ, Crane AM, Goldman-Rakic PS (1981) Direct and indirect pathways from the amygdala to the frontal lobe in rhesus monkeys. J Comp Neurol 198:121-136.

Powell DA, Maxwell B, Penney J (1996) Neuronal activity in the medial prefrontal cortex during Pavlovian eyeblink and nictitating membrane conditioning. J Neurosci 16:6296-6306.

Quirk GJ, Russo GK, Barron JL, Lebron K (2000) The role of ventromedial prefrontal cortex in the recovery of extinguished fear. J Neurosci 20:6225-6231.

Quirk GJ, Likhtik E, Pelletier JG, Paré D (2003) Stimulation of medial prefrontal cortex decreases the responsiveness of central amygdala output neurons. J Neurosci 23:8800-8807.

Radley JJ, Arias CM, Sawchenko PE (2006) Regional differentiation of the medial prefrontal cortex in regulating adaptive responses to acute emotional stress. J Neurosci 26:12967-12976.

Rajkowska G (2000) Postmortem studies in mood disorders indicate altered numbers of neurons and glial cells. Biol Psychiatry 48:766-777.

Rosenkranz JA, Grace AA (2001) Dopamine attenuates prefrontal cortical suppression of sensory inputs to the basolateral amygdala of rats. J Neurosci 21:4090-4103.

Servatius RJ, Shors TJ (1994) Exposure to inescapable stress persistently facilitates associative and nonassociative learning in rats. Behav Neurosci 108:1101-1106.

Shansky RM, Morrison JH (2009) Stress-induced dendritic remodeling in the medial prefrontal cortex: effects of circuit, hormones and rest. Brain Res 1293:108-113.

Shansky RM, Rubinow K, Brennan A, Arnsten AF (2006) The effects of sex and hormonal status on restraint-stress-induced working memory impairment. Behav Brain Funct 2:8.

Shors TJ (2004) Learning during stressful times. Learn Mem 11:137-144.

Shors TJ, Servatius RJ (1997) The contribution of stressor intensity, duration, and context to the stress-induced facilitation of associative learning. Neurobiol Learn Mem 68:92-96.

Shors TJ, Lewczyk C, Pacynski M, Mathew PR, Pickett J (1998) Stages of estrous mediate the stress-induced impairment of associative learning in the female rat. Neuroreport 9:419-423.

Sotres-Bayon F, Bush DE, LeDoux JE (2004) Emotional perseveration: an update on prefrontal-amygdala interactions in fear extinction. Learn Mem 11:525-535.

Takehara K, Kawahara S, Takatsuki K, Kirino Y (2002) Time-limited role of the hippocampus in the memory for trace eyeblink conditioning in mice. Brain Res 951:183-190.

Ter Horst GJ, Wichmann R, Gerrits M, Westenbroek C, Lin Y (2009) Sex differences in stress responses: focus on ovarian hormones. Physiol Behav 97:239-249.

Tolin DF, Foa EB (2006) Sex differences in trauma and posttraumatic stress disorder: a quantitative review of 25 years of research. Psychol Bull 132:959-992.

Vertes RP (2004) Differential projections of the infralimbic and prelimbic cortex in the rat. Synapse 51:32-58.

Vertes RP (2006) Interactions among the medial prefrontal cortex, hippocampus and midline thalamus in emotional and cognitive processing in the rat. Neuroscience 142:1-20.

Waddell J, Bangasser DA, Shors TJ (2008) The basolateral nucleus of the amygdala is necessary to induce the opposing effects of stressful experience on learning in males and females. J Neurosci 28:5290-5294.

Wood GE, Shors TJ (1998) Stress facilitates classical conditioning in males but impairs conditioning in females through activational influences of ovarian hormones. Proc Natl Acad Sci U S A 95:4066-4071.

Wood GE, Beylin AV, Shors TJ (2001) The contribution of adrenal and reproductive hormones to the opposing effects of stress on trace conditioning in males versus females. Behav Neurosci 115:175-187. 